\title{
Recuperação de Informações em Vídeos Educacionais: um estudo das funcionalidades semânticas.
}

\author{
Ismar F. Silveira, Paulo R. S. Borges \\ Programa de Pós-Graduação em Engenharia Elétrica (PPGEE) \\ Universidade Presbiteriana Mackenzie - São Paulo, SP - Brasil \\ ismar.silveira@mackenzie.br, 71412761 @mackenzista.com.br
}

\begin{abstract}
One of the great challenges on the use of educational videos is the research and recovery of video information. This study aims to build an educational semantic portal, specifically intended to educational audiovisual content. It seeks to identify, to list and to use tools that may help on the semantic cast in this content. Thus, it was designed an architecture proposal, that may amplify the possibilities on the recovery of content in educational videos.
\end{abstract}

Resumo. Um dos grandes desafios na utilização de vídeos educacionais é a pesquisa e recuperação de informações em vídeos. $O$ presente estudo tem por objetivo a construção de um portal semântico educacional, especificamente voltado a conteúdos audiovisuais educacionais. Pretende-se identificar, elencar e utilizar ferramentas que possam auxiliar na adição de semântica nesses conteúdos. Para tanto, foi delineada uma proposta de arquitetura, que pode ampliar as possibilidades de recuperação de conteúdos em vídeos educacionais.

\section{Introdução}

$\mathrm{Na}$ atualidade, a internet é utilizada para buscar as mais diversificadas informações que são acessadas em bancos on-line, lojas virtuais, comunidades virtuais, dentre muitas possibilidades oferecidas aos usuários. Essas informações estão presentes na web em diferentes formas, em motores de busca popular como o Google, de imagens como Yahoo, portais como YouTube, portais educacionais como Stanford on-line, por exemplo, que denunciam as elevadas taxas de produção de documentos multimídia produzidos e consumidos. Essa diversidade de informações implica em realização de descrições de conteúdo para o acesso, recuperação e reutilização dessas informações. Um gerenciamento inteligente de conteúdo pode auxiliar esse processo, mas exige a codificação semântica desses conteúdos (DASIOPOULOU et al, 2010).

A denominada web semântica ou web Inteligente ou web 3.0 surgiu nos anos 1990, quando Tim Berners-Lee idealizou a World Wide Web (WWW), uma modificação no conteúdo web, em decorrência da evolução de recursos web e da ampliação do número de usuários, pensando uma web com ampla interação entre usuário e internet. Ele pensou em estruturá-la de forma organizada por meio do acréscimo da semântica ao seu conteúdo, porém não foi possível com a tecnologia então disponível (BERNERS-LEE et al, 2001). 
Para Berners-Lee (2001), a maioria dos conteúdos disponíveis na web foi projetada com direcionamento aos usuários. O objetivo da web semântica é "trazer estrutura para o conteúdo das páginas web, aumentando-lhes o significado, criando um ambiente, em que agentes de software podem fluir de página a página, cumprindo sofisticadas tarefas para os seus usuários" (JORGE, 2005, p. 15). Segundo GarciaCastro et al ( 2008), na web semântica a reutilização não ocorre apenas no nível de dados, mas também no nível da aplicação, havendo muitos softwares que podem ser utilizados no desenvolvimento de aplicações semânticas. Para esses autores um framework web semântica consiste em uma estrutura para organizar e desenvolver aplicações da web semântica e é orientado por alguns princípios gerais de design, como: desenvolvimento orientado; fácil de entender; de simples utilização; focado em semântica visto que descreve somente os "componentes que fornecem funcionalidades semânticas e funcionalidades para gerenciar semântica" (GARCIA-CASTRO et al, 2008, p.201-202).

Nesse contexto se destacam os portais web, populares na Internet devido à sua capacidade na captura, organização e compartilhamento de informações integradas e estruturadas sobre determinado domínio. Os portais web tradicionais subsidiavam a troca de conteúdos entre os integrantes de grupos de indivíduos e se tornaram populares. Posteriormente emergiram os portais semânticos que utilizam os padrões de representação das informações da W3C tendo como base as ontologias que facilitam a organização das informações nesse ambiente (JORGE, 2005). E ainda contribuem para o compartilhamento e reutilização das informações contidas nesses portais e permitem arquiteturas mais sofisticadas de navegação, como também, respostas a consultas mais refinadas e que sejam estabelecidas inferências a partir de informações implícitas nos conteúdos (GRUBER, 1993).

Essas tecnologias que têm sido desenvolvidas para dar significado e promover a interação entre diversos sistemas auxiliam na adição de semântica em diversificados conteúdos disponibilizados aos usuários. A padronização do MPEG-7 no Multimedia Content Description Interface, por exemplo, foi desenvolvida no âmbito da comunicação de informação e reutilização com relação a documentos audiovisuais e fornece uma gama de instrumentos padronizados para o delineamento dos conteúdos audiovisuais em diversas "granularidades, abordando uma variedade de dimensões que variam de estrutural e de baixo nível, descrições para aspectos relacionados com a navegação, organização de conteúdos, bem como preferências do usuário e uso" (DASIOPOULOU et al, 2010, p.332). Esses conteúdos audiovisuais muitas vezes estão presentes em portais educacionais e pertencem a objetos de aprendizagem (OA) que consistem em recursos digitais reutilizáveis que são encapsulados em uma lição ou reunião de lições reunidas em unidades, módulos, cursos e mesmo programas (MCGREAL, 2004). A obtenção e recuperação das informações desses conteúdos podem ser alcançadas por meio das funcionalidades da web semântica.

Considerando esses pressupostos, em relação a vídeos educacionais presentes em Portais web educacionais, o estudo aqui proposto tem como hipótese que as funcionalidades semânticas poderiam facilitar o acesso às informações específicas constantes nesses vídeos, de acordo com o interesse dos usuários: professores e alunos. Assim, emerge as seguintes questões: como pode ser arquitetado um portal semântico educacional que seja asseado por material audiovisual? Quais as ferramentas no âmbito da comunicação, informação e reutilização, com relação a documentos audiovisuais, 
apresentadas na literatura, vêm sendo aplicadas para anexar semântica formal a conteúdos multimídia, especificamente destinados à educação?

Desse modo, o estudo aqui mencionado tem por objetivo a construção de um portal semântico educacional, especificamente voltado a conteúdos audiovisuais educacionais e ainda identificar, elencar e utilizar ferramentas que possam auxiliar na adição de semântica nesses conteúdos.

Este estudo está organizado da seguinte forma: a seção 2 refere à revisão da literatura. A seção 3 apresenta uma arquitetura de um portal semântico educacional. Por fim, as considerações finais deste trabalho são apresentadas na seção 4.

\section{Revisão da Literatura}

Estudos acerca dessa utilização de ferramentas semânticas têm sido desenvolvidos. Alan et al (2008) descreveu um sistema de anotação e consulta de vídeos apropriado para uma anotação semiautomática de vídeos a partir de texto. Alinhados com os segmentos de vídeo correspondentes os metadados extraídos permitiriam aos usuários a consulta de vídeos conforme o conteúdo semântico. Para demonstrar o uso do sistema, elegeram o domínio de futebol. Os vídeos anotados foram indexados com base em palavras chave, que permitiram a recuperação rápida de segmentos de vídeo. Guardaram em um banco de dados orientado a objetos no formato MPEG-7. A estrutura do sistema construído foi projetada de modo flexível para que as extensões preditas pudessem ser realizadas sem problemas e permitissem importar ontologias de diferentes domínios.

A investigação feita por Sebastine; Thuraisingham e Prabhakaran (2010) objetivou fornecer um motor de busca semântico em vídeo fundamentado na web. Apresentaram um quadro semântico baseado na web para a busca automática de recurso para armazenar, indexar e recuperar vídeos. Foram mapeados individualmente os vídeos sobre o seu arquivo RDF correspondente. Foi usado o sistema de arquivos do Google, para o armazenamento dos arquivos RDF. Indexaram os recursos existentes em todos os arquivos RDF com índices separados para cada tipo de dispositivo. A web semântica subsidia a recuperação de conteúdo baseado em multimídia e pode auxiliar na melhoria da qualidade da pesquisa de conteúdo.

O objetivo do estudo de Kanellopoulos (2012) foi propor um sistema de anotação semântica de objetos de mídia audiovisual decorridos no domínio de um documentário e discutir uma abordagem de evolução de rede semântica para a descrição do conteúdo audiovisual. Apresentou como um documentário de mídia poderia ser anotado semanticamente e como a informação poderia ser empregada para a obtenção dos objetos de mídia. Considerou que atualmente uma organização maleável da mídia solicita a construção de uma rede semântica, mas reconheceu que a abordagem é suscetível a mudanças e provê técnicas estruturais de memória. Evidenciou que a abordagem proposta pode facilitar o uso de material audiovisual de modo dinâmico e permitir conexão semântica entre os nós de informação usando relações datilografadas, estruturando o espaço de informação em um nível semântico e sintático.

Lachtim (2008) propôs o uso de ontologias que auxiliassem a alimentação de portais semânticos. Pretendeu formular consultas para um mecanismo de busca na web fundamentada na ontologia de domínio que subsidia o portal, com o auxilio de um dicionário de termos. A partir dos resultados encontrados, esse dicionário auxilia a 
formação de uma visão integrada das informações, o que permite a organização e publicação dinâmica no portal. Para a ilustração da sistemática proposta, esse trabalho apresentou uma ontologia de domínio criada especialmente para um Portal Semântico Educacional.

A investigação de Siqueira et al (2008) propôs uma segmentação semântica do conteúdo incorporado em OA existente, organizando-o em segmentos semânticos que podem ser reutilizados. Essa segmentação segue uma conceituação todo-parte e a implementação desses segmentos admite suas respectivas descrições por meio de agrupamentos complementares de metadados. Utilizaram a ferramenta de estruturação semântica que possibilita ao usuário: uma análise do conteúdo contido nos OA, segmentá-los e descrever os segmentos obtidos. Posteriormente estruturaria novos OA a partir de diversificados segmentos semânticos. Em uma arquitetura de segmentação multimídia OA, partes do MPEG-7 foram utilizadas em um estudo de caso e um protótipo construído, os quais apresentaram a funcionalidade da arquitetura proposta. Observaram que da ideia de reuso aplicada sobre segmentos de OA decorre uma ampliação no potencial de reutilização desses objetos.

Silva e Gluz (2012) objetivaram desenvolver uma ferramenta que propiciasse mecanismos de busca semântica de objetos de aprendizagem com suporte a alinhamento de ontologias. Concluíram que as técnicas referentes ao alinhamento de ontologias ainda estão em um estágio inicial, reconhecem a complexidade a ser enfrentada e pleitearam ter contribuído para a otimização do processo de alinhamento de ontologias. Já Patrocinio e Ishitani (2009) descreveram uma proposta de um modelo de dados para a anotação de OA presentes em repositórios heterogêneos. Foram usados metadados de padrões estabelecidos e a associação de recursos de anotação colaborativa e uma aplicação cliente que usa o modelo proposto. A associação desses recursos amplia as possibilidades de busca e navegação por objetos com o uso de etiquetas, o que auxilia a integração de repositórios heterogêneos.

Esses estudos mostram que as tecnologias digitais de comunicação e informação estão presentes nos mais variados segmentos, dentre as quais os OA, no âmbito educacional. Analogamente ao modelo da programação orientada a objetos, os OA construídos e detentores de anotação semântica adequada podem ser guardados em repositórios de modo a serem reutilizados e referenciados no meio educacional. Assim, o desenvolvimento de novas tecnologias pode auxiliar na melhoria dos processos de localização, catalogação, recuperação e reutilização de informações contidas nesses OA, especificamente em vídeos educacionais que estão em portais semânticos educacionais.

Nos portais semânticos a pesquisa é multidimensional por significado de uma ontologia rica de domínio (REYNOLDS; SHABAJEE; CAYZER, 2004). Além disso, promovem a organização das informações, de forma mais avançada, subsidiados pelas tecnologias da web semântica. Uma de suas funcionalidades é a personalização que adapta as ferramentas desses portais às necessidades dos usuários que os utilizam para acessar as informações. Nesse sentido, os mecanismos de busca têm se destacado como ferramentas úteis no fornecimento de resultados eficientes em curto espaço de tempo. A categorização de assuntos é outra funcionalidade que busca a simplificação da navegação, adicionando informações essenciais na classificação dos documentos, para que sejam organizados em conformidade com uma ontologia (JORGE, 2005). 
Para o delineamento dos conteúdos audiovisuais, o MPEG-7 oferece uma grande quantidade de instrumentos padronizados. A ontologia do MPEG-7 proposta por Hunter em 2001 é considerada a primeira ação objetivando anexar semântica formal a MPEG7. Foi proposta a linguagem RDF Schema (RDFS) intentando formalizar os descritores contidos na parte visual e a estrutura e as ferramentas de localização do MPEG-7 MDS. Ainda foi incluído um conjunto de descritores referentes às informações acerca das características de produção, criação, uso e mídia. Para Heinzle (2011), a construção de ontologias se fundamenta em algumas linguagens formais específicas que se distinguem pelas facilidades e propriedades computacionais que possuem, quais sejam: RDF; RDFSchema, OIL (Ontology Inference Layer), DAML (DARPA Agent Markup Language) OIL e OWL (Ontology web Language) (DASIOPOULOU et al, 2010).

Em uma representação ontológica, a interpretação das definições do MPEG-7 adere ao esquema original MPEG-7. Nesse processo, são modelados como classes os diversificados conteúdos de MPEG-7 e tipos de segmento. Desse modo, enquanto propriedades têm sido usadas para modelar os esquemas de decomposição, conservando a flexibilidade propiciada pelo MPEG-7, diferentes tipos de segmento são abordados como tipos de conteúdos de multimídia. Além disso, adota-se que suas inferências podem resultar de um pequeno conjunto de sentenças lógicas, e que as entidades semânticas podem compartilhar um mesmo significado semântico. Em decorrência, as ambiguidades do MPEG-7 são replicadas, incidindo em implicações na nitidez conceitual e na gestão posterior das descrições produzidas (DASIOPOULOU et al, 2010).

\section{Arquitetura de um portal semântico educacional asseado em material audiovisual}

Nesta seção é apresentada a arquitetura proposta para a construção de um portal semântico educacional que conterá um repositório com vídeos educacionais, organizados de acordo com o nível de dificuldade e categoria. Essa arquitetura será constituída por vários módulos. No entanto, destacar-se-á somente a funcionalidade de edição e obtenção de conteúdo de vídeos educacionais, de acordo com o objetivo deste estudo.

Para estruturar o Portal Semântico Educacional como proposto neste estudo, utilizar-se-á do padrão MPEG-7, o qual é estruturado em partes distintas, quais sejam: linguagem de definição de descrição, a DDL; visual, o conjunto composto por descritores e esquemas de descrição de conteúdos visuais; áudio, conjunto composto por descritores e esquemas de descrição de conteúdos áudio; Esquema de descrição multimídia, o MDS (MATUSHIMA, 2007).

Os MDS podem ser agrupados em cinco classes diferenciadas de acordo com a sua funcionalidade. A primeira, descrição do conteúdo antecipa mecanismos de descrição da semântica e da estrutura de um conteúdo audiovisual, podendo ser realizada em diversificados níveis e estar relacionada a um segmento do vídeo, a uma determinada cena ou ainda a uma ação especifica ou região de uma cena (MATUSHIMA, 2007). Nessa direção a presente proposta de arquitetura contemplará um editor, que possibilitará realizar anotações semânticas e visuais nesses vídeos.

Em relação ao gerenciamento de conteúdo, conforme proposto por Matushima (2007), possibilita a descrição de informações relativas à mídia do conteúdo, dados do 
processo de sua produção e informações de identificação. Nesse sentido, a presente proposta de arquitetura permitirá ao usuário a administração de conteúdos de vídeos e meta-dados através de um gerenciador de conteúdo on-line (CMS - Content management system) que será implementado, levando em consideração a experiência do usuário.

No que tange a organização do conteúdo, segundo Matushima (2007), envolve esquemas de descrição de propriedades comuns conjuntos de conteúdos multimídia, segmentos de informação, eventos e objetos, com fins de organizá-los. Assim, a presente proposta de arquitetura viabilizará aos usuários, o acesso e recuperação das informações com base em uma ontologia de domínio e categorizações.

Em relação à navegação e acesso, o MPEG-7 fornece esquemas de descrição e descritores que auxiliam o processo de busca de conteúdo audiovisual e de navegação (MATUSHIMA, 2007). Analogamente a esse estudo, esta proposta de arquitetura contará com um gerenciador de usuários que possuirão uma área destinada à pesquisa e à navegação nos conteúdos dos vídeos educacionais disponíveis nesse portal.

Com referência à interação com o usuário, determina a utilização do material multimídia (MATUSHIMA, 2007). Neste sentido, esta proposta de arquitetura de um portal semântico educacional asseado em material audiovisual possuirá diferentes perfis para usuários, com o intuito de gerenciar as funcionalidades possíveis para cada grupo de usuário. $\mathrm{O}$ usuário administrador terá permissão para gerenciar os vídeos e seus meta-conteúdos. Já o usuário com perfil de visualizador poderá apenas buscar e acessar os conteúdos semanticamente.

Em decorrência dos estudos realizados são apresentadas as ferramentas e ou tecnologias para a construção da arquitetura aqui proposta, por permitirem agregar novas funcionalidades aos conteúdos multimídia: o framework Spring que fornece um modelo abrangente de programação e configuração de aplicativos corporativos baseados em Java (http://projects.spring.io/spring-framework/) que irá conter os serviços responsáveis pela lógica de negócio do portal e ficará responsável pela interface entre a camada de apresentação e de persistência; a Tecnologia JavaServer Faces- JSF que institui um padrão para a construção de interfaces com o usuário do lado do servidor (http://www.oracle.com/technetwork/java/javaee/javaserverfaces-139869.htmltO) o que torna o desenvolvimento mais rápido; as bibliotecas de JavaScript e Alloy que fornecem uma API simples para construir aplicações escalonáveis (http://alloyui.com/); D3.js que permite a manipulação de documentos com base em dados. D3 auxilia exibir informações aos usuários usando HTML, SVG e CSS (http://d3js.org/). Ainda, o HTML5 e CSS3 (http://www.w3.org/TR/html5/) que permitirão a criação das interfaces gráficas de usuário; o framework JPA com a implementação em Hibernate que permite aos desenvolvedores escrever aplicativos de modo mais fácil (http://hibernate.org/) e o Jena, framework de código livre e aberto para construção de aplicativos Linked Data (https://jena.apache.org/) que constituirão a camada de persistência; o sistema de gerenciamento de banco de dados Oracle $10 \mathrm{~g}$ que armazenará as informações de todo o portal(oracle.com); o servidor web Apache Tomcat que irá hospedar o portal (http://tomcat.apache.org/); e o ambiente de desenvolvimento integrado Eclipse(eclipse.org). Assim, a arquitetura dessas ferramentas e/ou tecnologias será como apresentada na Figura 1. 


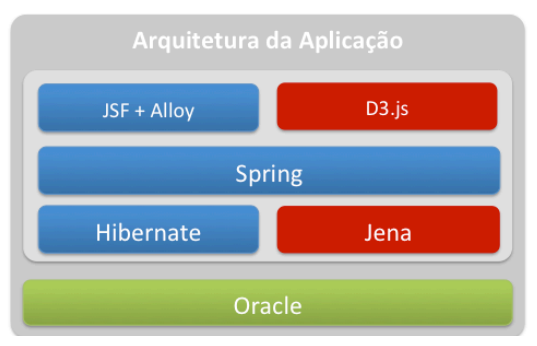

Figura 1. Arquitetura Proposta

Dentre as tecnologias já elencadas, na arquitetura aqui proposta, será utilizado o Jena, de código aberto escrito em Java para web semântica de modo a realizar a gravação e a recuperação de RDF graphs. Essa ação permite realizar consultas do tipo SPARQL para a obtenção das informações. Além disso, esse framework oferece suporte à OWL. Essa escolha foi fundamentada em Sebastine; Thuraisingham e Prabhakaran (2010, p.104) que usaram o Jena, um framework Java comumente usado em web semântica. Para esses autores a comunidade web semântica tem utilizado amplamente RDF para indexar dados texto.

Para o desenvolvimento do portal semântico educacional, será utilizado o processo de desenvolvimento interativo e incremental ágil SCRUM com a finalidade de garantir a satisfação do usuário desse portal e sua rápida adaptação às mudanças necessárias (scrum.org).

$\mathrm{Na}$ arquitetura aqui proposta optou-se por utilizar como base as ontologias MPEG-7 apresentadas por esses autores. Para Kanellopoulos (2012, p. 743) as "ontologias multimídia (ontologias especialmente baseados em MPEG-7) tem o potencial de aumentar a interoperabilidade das aplicações produtores e consumidores anotações multimídia”. Dasiopoulou et al (2010) utilizaram ontologias que foram construídas manualmente na linguagem OWL, um padrão do W3C para a Web, por ser uma linguagem de representação usualmente selecionada. Para esses autores, essas ontologias seguem uma arquitetura modular, o que facilita "a separação de interesses, extensibilidade e uma gestão eficaz da produção de metadados" (DASIOPOULOU et al, 2010, p.342).

Para Alan et al (2008), os usuários desejam pesquisar e encontrar conteúdos audiovisuais, rapidamente, levando em conta a semântica desses conteúdos, com foco em um ponto certo do vídeo apenas por meio de busca com texto livre. Para tanto é necessário que haja conhecimento sobre o conteúdo, o qual vem a partir dos metadados armazenados como anotações juntamente com os vídeos. Pensando por essa lógica, arquitetou a página inicial do portal semântico educacional proposto neste estudo, a qual será construída e nela disponibilizada uma área de login e cadastramento de usuários. Esse cadastro irá conter os seguintes campos: nome de usuário, senha, idade e áreas de interesse. Uma vez cadastrado, o usuário poderá usufruir de todas as funcionalidades desse portal, relacionadas ao seu perfil. Além disso, essa página irá conter os vídeos em destaque e um campo para a busca semântica. A figura 2 mostra essa página inicial. 


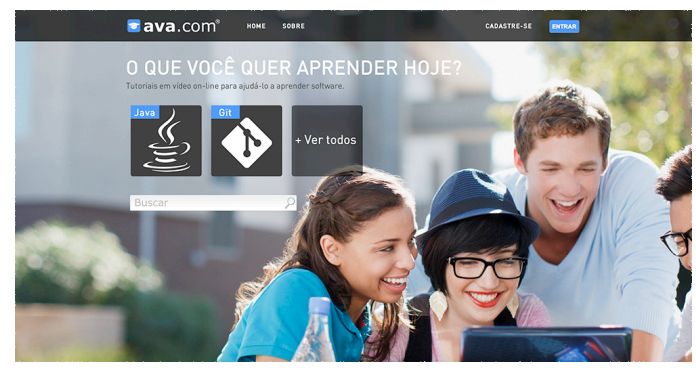

Figura 2. Página inicial do portal

Em relação à busca, esse portal semântico educacional oferecerá dois tipos de pesquisa: uma simples, em que será exibida apenas uma caixa de texto, a qual permitirá realizar uma busca em todos os vídeos educacionais constantes no repositório desse portal; e outra avançada, disponibilizando vários filtros que auxiliarão em uma pesquisa mais específica, quais sejam: data de criação, data de atualização, número de visualizações, autor, nível de dificuldade e categoria.

Alan et al (2008) proveem uma funcionalidade de pesquisa que mostra os resultados da busca semântica em vídeos de futebol. Os resultados das consultas foram apresentados instantaneamente no início do segmento de vídeo em que é encontrada a palavra chave. Cada parte do arquivo de anotações e índice possui um arquivo de imagem que representa essa parte do vídeo. Dessa forma, o arquivo de imagem do segmento correspondente é um campo no índice da base do sistema, mas esse índice só armazena a referência da imagem. Tomando novamente por base os estudos de Alan et al (2008) para a construção da arquitetura proposta, será propiciada uma busca em forma de segmento, entretanto, a exibição dos conteúdos encontrados pela busca nesse portal, se dará em forma de listagem e em forma de árvore hiperbólica, a qual permitirá uma navegação mais interativa por parte do usuário. A figura 3 apresenta essa página do portal disponibilizada com o acesso do usuário.

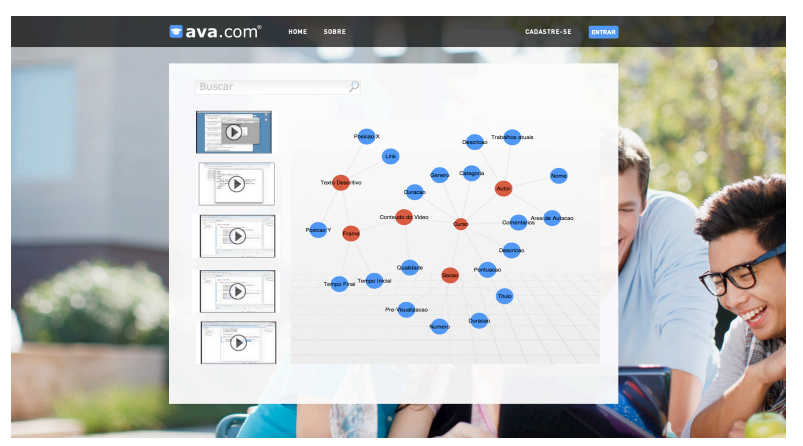

Figura 3. Resultado de buscas no portal

A principal característica do sistema desenvolvido por Alan et al (2008) é a permissão para a busca dos usuários dentro dos arquivos de vídeos. Como um resultado de pesquisa do usuário, as cenas almejadas do arquivo de vídeo devem ser recuperadas. Considerando que algumas perguntas feitas pelo usuário podem ser respondidas por pesquisa com palavras-chave, contudo são desperdiçadas algumas cenas ou vídeos, os autores utilizaram busca semântica com a finalidade de aumentar a qualidade das ferramentas de pesquisa do sistema. Esse desenvolvimento influenciou na escolha das ferramentas utilizadas nesta proposta de construção do portal semântico educacional, de modo que os usuários, ao clicarem no vídeo educacional escolhido, serão redirecionados 
para a tela de detalhamento do conteúdo do vídeo, juntamente com os metadados de MPEG-7, associados a segmentos semânticos obtidos do vídeo.

$\mathrm{Na}$ arquitetura do portal semântico educacional aqui proposto haverá uma tela em que os usuários que possuem perfil para o gerenciamento de vídeos, poderão realizar a criação do conteúdo semântico pela interface da ferramenta de edição de conteúdo. Para tal, serão utilizados componentes para o preenchimento dos metadados associados ao padrão MPEG-7 com a finalidade de criação do conteúdo semântico, juntamente com um pré-visualizador para a análise desse conteúdo. Essa escolha se baseia no estudo de Alan et al(2008) no qual utilizaram uma ferramenta de anotação desenvolvida para esta finalidade que permite a anotação manual de usuários sobre o conteúdo de um vídeo e gera dinamicamente os campos de dados de acordo com o domínio.

\section{Considerações finais}

A revisão da literatura acerca do tema em estudo permitiu conhecer os métodos usados atualmente para incluir semântica em vídeos disponíveis na web e analisar a eficiência desses estudos de acordo com os objetivos alcançados. Além disso, permitiu estabelecer um cenário da aplicação dos conceitos de web semântica para incluir semântica em vídeos disponíveis na web sob condições controladas. Os resultados desses estudos apresentaram que foram criados sistemas que admitiram importar ontologias de diferentes domínios que podem acrescentar semântica nos conteúdos multimídia, integrar e recuperar conteúdos base, organizar documentário mídia, construir uma rede semântica e permitir a reutilização e a extensibilidade da ferramenta construída.

Esses pressupostos teóricos fundamentaram a proposta em desenvolvimento de uma arquitetura de um portal semântico educacional asseado em material audiovisual, que embora ainda inicial, já traz um escopo das ferramentas que estão sendo utilizadas e um panorama do que será propiciado nesse portal para que os usuários possam ter acesso aos conteúdos desses vídeos com autonomia. Este estudo permite afirmar que as funcionalidades semânticas podem auxiliar na recuperação de conteúdos em vídeos educacionais em tempo real, o que propicia a integração desses conteúdos e consequentemente uma melhoria na qualidade dos resultados e eficiência das pesquisas feitas pelos usuários.

Trabalhos futuros apontam para a realização de testes com usuários finais de maneira a validar adequadamente a eficácia do uso de mecanismos semânticos na recuperação de conteúdos audiovisuais educacionais.

\section{Referências}

ALAN, O. et al. Ontological Video Annotation and Querying System for Soccer Games. [S.1.: s.n.], 2008. 431-436 p. 23rd International Symposium on Computer and Information Sciences, Istanbul, TURKEY, OCT 27-29, 2008. ISBN 978-1-42442880-9.

BERNERS-LEE, T.; HENDLER, J.; LASSILA, O. The Semantic Web - A new form of Web content that is meaningful to computers will unleash a revolution of new possibilities. [S.1.: s.n.], 2001.34+ p. ISSN 0036-8733.

DASIOPOULOU, S. et al. Enquiring MPEG-7 based multimedia ontologies. [S.l.: s.n.], 2010. 331-370 p. ISSN 1380-7501. 
GARCÍA-CASTRO, R. et al. Towards a component-based framework for developing Semantic Web applications. In: The Semantic Web. Springer Berlin Heidelberg, 2008. p. 197-211.

GRUBER, T. R. Toward principles for the design of ontologies used for knowledge sharing? [S.1.]: Elsevier, 1993. 907-928 p.

HEINZLE, R. Um modelo de engenharia do conhecimento para sistemas de apoio a decisão com recursos para raciocínio abdutivo. [S.l.: s.n.], 2011.

JORGE, M. A. Ontologias no Suporte a Portais Semânticos. Dissertação de Mestrado apresentada ao Programa de Pós-Graduação da Escola de Ciência da Informação da Universidade Federal de Minas Gerais: [s.n.], 2005. 15 p. http://www.bibliotecadigital.ufmg.br/dspace/bitstream/1843/VALA-6KFNS2/1/ mestrado__marcelo_alvim_jorge.pdf/. [Online; Acesso em: 17-dez-2011].

KANELLOPOULOS, D. Semantic annotation and retrieval of documentary media objects. [s.n.], 2012. 721-747 p. Disponível em: <http://www.emeraldinsight.com/doi- /abs/10.1108/02640471211275756>.

LACHTIM, F. A. et al. Uma Abordagem Baseada em Ontologias para a Busca de Conteúdos na Web e Publicação em Portais Semânticos. [S.1.: s.n.], 2009.

MATUSHIMA, R. Desenvolvimento de aplicações multimídia baseado em arquitetura orientada a serviços e nos padrões MPEG-7 e MPEG-21. 2007. Dissertação (Mestrado em Sistemas Digitais) - Escola Politécnica, Universidade de São Paulo, São Paulo, 2007. Disponível em: $<$ http://www.teses.usp.br/teses/disponiveis/3/3141/tde-08012008-150321/>. Acesso em: 2014-10-12.

MCGREAL, R., et al. (2004). EduSource: Canada's Learning Object Repository Network. The International Journal of Instructional Technology and Distance Learning, 1(3). Disponível em: http://www.itdl.org/Journal/Mar_04/article01.htm. Acesso: 15 out. 2014.

PATROCINIO, M.; ISHITANI, L. Associação de Recursos Semânticos para a Anotação de Objetos de Aprendizagem. [S.1.: s.n.], 2012.

REYNOLDS, D.; SHABAJEE, P.; CAYZER, S. Semantic information portals. [S.1.], 2004. 290-291 p.

SEBASTINE, S. C.; THURAISINGHAM, B.; PRABHAKARAN, B. Semantic Web for Content Based Video Retrieval. [S.1.], 2009. 103-108 p. 3rd International Conference on Semantic Computing (ICSC 2009), Berkeley, CA, SEP 14-16, 2009. ISBN 978-14244-4962-0. 